\title{
Successful Implantable Cardioverter Defibrillator Implantation in a Patient with Persistent Left Superior Vena Cava and Atrial Septal Defect
}

Ki Hong Lee, MD; Hyung Wook Park, MD; Nam Sik Yoon, MD; Sung Soo Kim, MD; Jeong Gwan Cho, MD

The Heart Research Center of Chonnam National University Hospital, Cardiovascular Research Institute of Chonnam National University, Gwangju, Korea

Received: January 13, 2015

Revision Received: June 1, 2015

Accepted: June 29, 2015

Correspondence: Hyung Wook Park, MD, PhD, Associate Professor, Cardiovascular Medicine, Chief of Cardiovascular Medicine, The Heart Center of Chonnam National University Hospital, 42 Jaebongro, Dong-gu, Gwangju 501-757, South Korea

Tel: +82-62-220-6572, Fax: +82-62-223-3105

E-mail:mdhwp@chol.com

Copyright (c) 2015 The Official Journal of Korean Heart Rhythm Society Editorial Board \& MMK Co., Ltd.

\begin{abstract}
Use of an implantable cardioverter defibrillator (ICD) is the treatment of choice in patients experiencing sudden cardiac arrest. However, the presence of persistent left superior vena cava (PLSVC) presents technical challenges and complicates the ICD implantation because of the high frequency of accompanying congenital anomalies. We report on a right-handed 34 year-old male patient with PLSVC and atrial septal defect undergoing successful ICD implantation in the left anterior chest. The patient had been successfully resuscitated from idiopathic ventricular fibrillation. Computed tomography demonstrated dual SVC with a rudimentary cross-connection. However, the guide-wire did not cross the innominate vein. Therefore, the placement of an ICD lead at the right atrium over the huge coronary sinus was decided. Access to the right ventricle was gained by using a hand-shaped r-shaped stylet, with its distal end perpendicular to the proximal part. An ICD lead was positioned at the right ventricular apex with satisfactory measurement.
\end{abstract}

Key Words: - Defibrillators, Implantable -Vena Cava, Superior - Heart Septal Defects, Atrial

\section{Introduction}

Use of an implantable cardioverter defibrillator (ICD) is the treatment of choice in patients with aborted sudden cardiac arrest. Device implantation and venous access in the left anterior chest is usually used in right-handed patients because this site is opposite to the dominant hand. The presence of persistent left superior vena cava (PLSVC) can complicate lead placement within the right side of the heart. PLSVC is a rare vascular anomaly, present in 0.3 to $2 \%$ of the population. ${ }^{1}$ It also indicates a 10 -fold higher incidence of congenital cardiac anomalies. ${ }^{2}$ Therefore, implantation of a cardiac device using the right anterior chest approach is preferred in patients with PLSVC. Although implantation of a cardiac device in the left anterior chest is more comfortable in right-handed patients, this presents technically challenging situations because it complicates the ICD implantation procedure. Here we report on a right-handed patient with PLSVC and atrial septal defect (ASD) undergoing successful ICD implantation in the left anterior chest. 

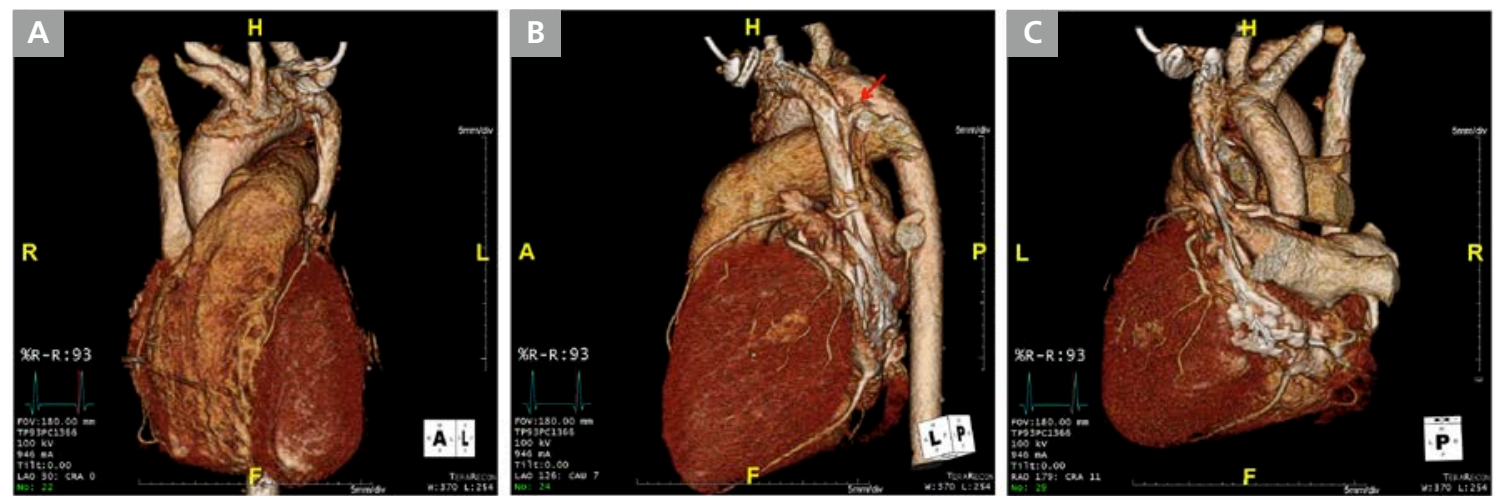

Figure 1. Cardiac computed tomography. (A) Anterior-posterior view showed double superior vena cava. (B) Left posterior oblique view demonstrated rudimentary innominate vein (arrow). (C) Posterior-anterior view demonstrated persistent left superior vena cava draining into the huge coronary sinus.

A

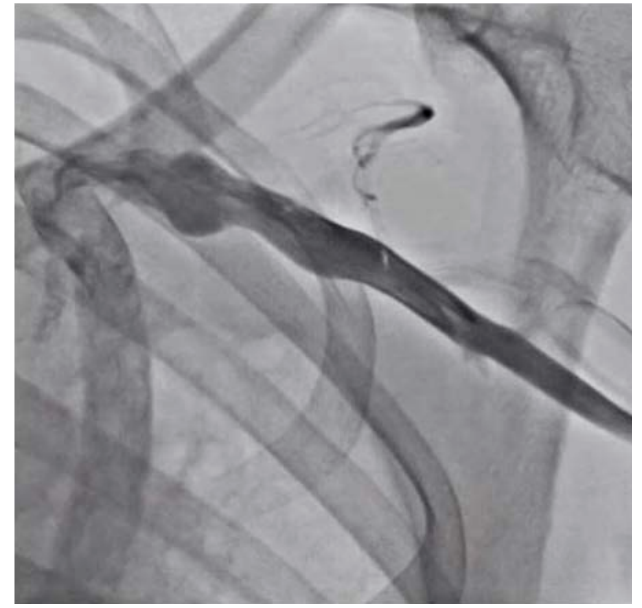

B

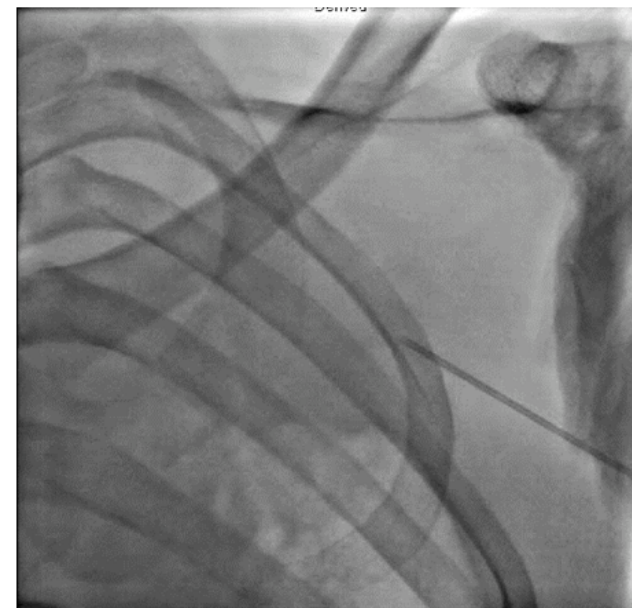

Figure 2. A venous angiography. (A) A venous angiography at the left antecubital vein demonstrated a persistent left superior vena cava. (B) A hydrophilic wire (Radiofocus ${ }^{\circledast}$ Guide Wire M, Terumo ${ }^{\oplus}$, Somerset, USA) was advanced into the persistent left superior vena cava via a left axillary vein puncture parallel to venous drainage.
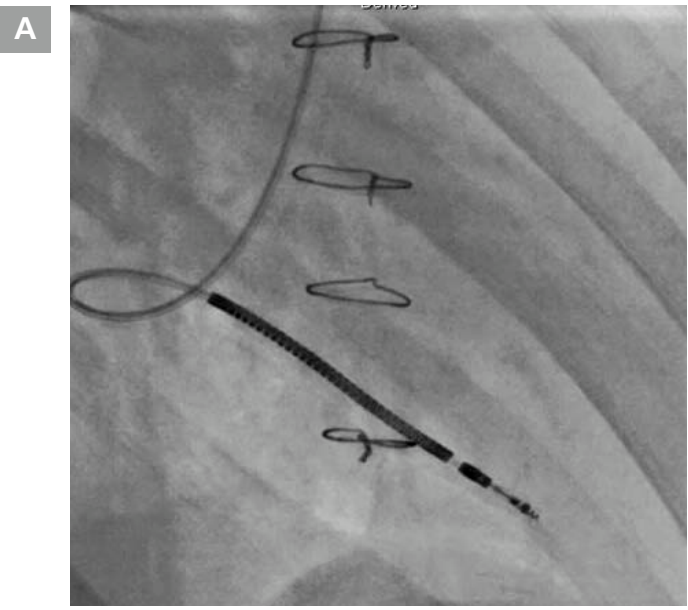

B

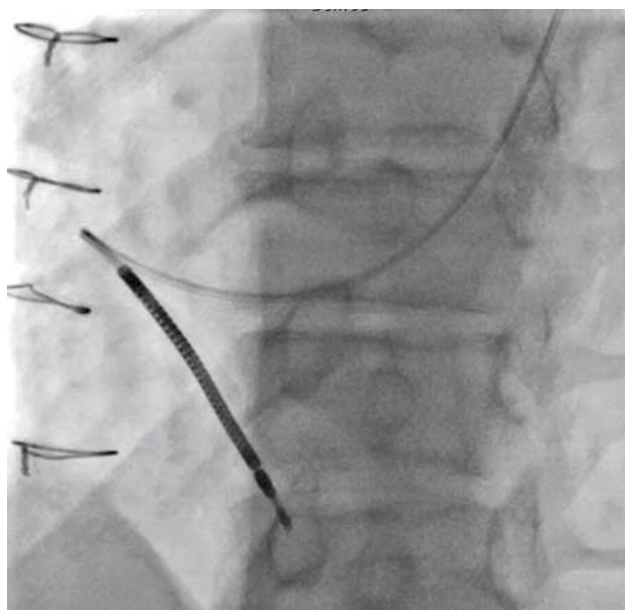

Figure 3. Fluoroscopic image of the final ventricular lead position. (A) Anterior-posterior view. (B) Left anterior oblique view. 


\section{Case}

A 34 year-old male patient presented to the emergency department undergoing cardiopulmonary resuscitation (CPR). He had suddenly collapsed during smartphone manipulation. The emergency medical service team activated the automatic electrical defibrillator (AED) as they diagnosed ventricular fibrillation. The team transferred the patient while performing CPR. The patient had undergone surgical correction of ASD 20 years previously, and was being treated for multidrug resistant pulmonary tuberculosis for 2 years. Therapeutic hypothermia aided recovery from hypoxic brain damage after return of spontaneous circulation. A detailed evaluation was performed to identify the cause of sudden cardiac arrest and included coronary angiography, echocardiography, laboratory examination, the flecainide provocation test for unmasking Brugada syndrome, and the epinephrine challenge test for unmasking long QT syndrome. Echocardiogram revealed the presence of PLSVC without any other structural cardiac anomaly. A contrast-enhanced computed tomography was performed to assess anatomic structures and their relationship to any combined anomaly. Computed tomography showed a double superior vena cava (SVC) with a rudimentary cross-connection and a faint trace of the surgical correction of ASD (Figure 1). The patient was diagnosed as having idiopathic ventricular fibrillation with evidence of past surgical correction of ASD. To prevent further cardiac arrest, ICD implantation was planned.

A venous angiography via the left antecubital vein revealed PLSVC drainage into the huge coronary sinus without visualization of any cross-connection with right side SVC (Figure 2A). A hydrophilic wire (Radiofocus ${ }^{\circledR}$ Guide Wire M, Terumo, Somerset, USA) was advanced into the PLSVC via a left axillary vein puncture parallel to venous drainage (Figure $2 \mathrm{~B}$ ). In a prior trial of left axillary venous puncture at a more distal location, an n-shaped acute angle did not allow peel-away sheath advancement. Although the rudimentary innominate vein was visualized on computed tomography, a hydrophilic wire did not cross. Subsequently, a single coil ICD lead (DURATA, 7122Q-58 $\mathrm{cm}$, active fixation, St Jude Medical) was introduced into the right atrium via the huge coronary sinus. Access to the right ventricle was gained by using a hand-shaped r-shaped stylet, its distal end perpendicular to the proximal part. The ICD lead was rotated counterclockwise directly towards the tricuspid annulus (Figure 3) and positioned at the right ventricular apex with satisfactory measurement.

\section{Discussion}

ICD implantation in patients with PLSVC generates technical challenges due to differences in anatomy including congenital abnormalities. However, a sufficient understanding of anatomy helps in planning the procedure and preparing suitable materials, leading to successful implantation.-5 Additionally, operators should be aware of combined congenital anomalies that accompany PLSVC. ${ }^{2}$ This patient had a medical history of surgical ASD correction. Transthoracic and trans-esophageal echocardiography and computed tomography confirmed that this patient did not have any other structural anomaly. The major difficulty is locating the ventricular lead across from the tricuspid annulus. Previous studies suggest several possible methods: (a) reshaping the stylet manually into a U-shape, (b) utilizing a preformed J-shaped stylet, (c) forming a loop in the right atrium using the right atrial free wall for support, and (d) placing the ventricular lead in the left ventricular branch of the coronary sinus. ${ }^{1,4,6}$ In the present study, an r-shaped stylet was used instead of the traditional J- or U-shaped stylet. The r-shaped stylet was chosen because this shape was directed toward the tricuspid annulus, making it easier to advance into the right ventricle from the coronary sinus ostium. In summary, understanding complex anatomy, planning with active detailed discussions, and having suitable materials available enables successful cardiac device implantation in patients with PLSVC.

\section{References}

1) Biffi M, Boriani G, Frabetti L, Bronzetti G, Branzi A. Left superior vena cava persistence in patients undergoing pacemaker or cardioverter-defibrillator implantation: A 10-year experience. Chest. 2001;120:139-144.

2) Paval J, Nayak S. A persistent left superior vena cava. Singapore Med J.2007;48:e90-93.

3) Cagin C, Barsness GW, Friedman PA, Cha YM. Coronary 
venoplasty-assisted implantation of cardiac resynchronization device in a patient with persistent left superior vena cava. Heart Rhythm. 2010;7:141-142.

4) Guenther M, Kolschmann S, Rauwolf TP, Christoph M, Sandfort V, Strasser RH, Wunderlich C. Implantable cardioverter defibrillator lead implantation in patients with a persistent left superior vena cava--feasibility, chances, and limitations: Representative cases in adults. Europace. 2013;15:273-277.

5) Konstantino Y, Kusniec J, Shohat-Zabarski R, Battler A, Strasberg B. Cardiac defibrillator implantation via persistent left superior vena cava facilitated by a coronary sinus delivery system. Europace. 2009;11:119-120.

6) Paperini L, Pardini E, Ebert AG, Galli M. Transvenous cardioverterdefibrillator implantation with a double coil lead via persistent left superior vena cava. Ital Heart J. 2004;5:711-713. 